\title{
Optical cavity modes in gold shell colloids
}

\author{
J. J. Penninkhof, ${ }^{1}$ L. A. Sweatlock, ${ }^{2}$ A. Moroz, ${ }^{3, b)}$ H. A. Atwater, ${ }^{2}$ A. van Blaaderen, ${ }^{3, c)}$ \\ and A. Polman ${ }^{1, a)}$ \\ ${ }^{1}$ Center for Nanophotonics, FOM Institute for Atomic and Molecular Physics (AMOLF), Kruislaan 407, \\ 1098 SJ Amsterdam, The Netherlands \\ ${ }^{2}$ Thomas J. Watson Laboratory of Applied Physics, California Institute of Technology, Pasadena, California \\ 91125, USA \\ ${ }^{3}$ Debye Institute for Nanomaterials Science, Utrecht University, P.O. Box 80000, 3508 TA Utrecht, \\ The Netherlands
}

(Received 7 January 2008; accepted 3 April 2008; published online 18 June 2008)

\begin{abstract}
Core-shell colloids composed of a dielectric core surrounded by a metal shell show geometric cavity resonances with optical properties that are distinctly different than those of the collective plasmon modes of the metal shell. We use finite-difference time domain calculations on silica colloids with a core diameter of $456 \mathrm{~nm}$, surrounded by a $38 \mathrm{~nm}$ thick Au shell, to study the temporal evolution of the mode field intensity inside the cavity upon pulsed excitation. Calculations using Mie theory and the $T$-matrix method are used to analytically determine the dipolar cavity resonance spectrum, which is found superimposed on the broad collective dipolar plasmonic resonance modes. We characterize resonance wavelength and linewidth in terms of a geometric mode confined inside the cavity. Cavity linewidth can be controlled by metal shell thickness and quality factors $Q>150$ are observed. Due to the small cavity mode volume $V=0.2(\lambda / n)^{3}$, a Purcell factor as high as $P=54$ is calculated. Introducing shape anisotropy lifts the cavity mode degeneracy, yielding blue- and redshifted longitudinal and transverse resonant modes, respectively. The relatively large volume over which the field enhancement is observed in these spherical and anisotropic metal shell cavities, combined with cavity quality factors that are much higher than that of the collective plasmonic modes, makes them attractive for application in nanoscale light sources, sensors, or lasers.
\end{abstract}

(C) 2008 American Institute of Physics. [DOI: 10.1063/1.2939249]

\section{INTRODUCTION}

Small (noble-) metal particles exhibit interesting optical properties due to the collective oscillation of conduction electrons (surface plasmons). This localized surface plasmon mode causes confinement of the electromagnetic field near the surface and leads to strong absorption and scattering in the visible and near infrared, depending on the geometry, size, and shape of the metal particle. The enhanced local fields can be used to enhance the fluorescence emission of dyes close to the metal surface, ${ }^{1,2}$ to give increased signals in Raman spectroscopy, ${ }^{3,4}$ or to increase the photostability of luminescent dyes by shortening the excited-state lifetime. ${ }^{5-9}$

An interesting class of metal nanoparticles are core-shell colloids, composed of a dielectric core surrounded by a metallic shell. The plasmon frequency of these particles can be tuned throughout the visible and near-infrared part of the spectrum by varying core diameter and shell thickness. ${ }^{10-12}$ Additional tunability can be obtained by shape anisotropy, which induces a splitting of the plasmon resonance in redshifted longitudinal and blueshifted transverse plasmon bands. ${ }^{13-16}$ Strong surface-enhanced Raman signal enhancement was observed in spherical core-shell colloids, ${ }^{4,17,18}$ and finite-difference time domain (FDTD) calculations ${ }^{19}$ as well

\footnotetext{
${ }^{a)}$ Electronic mail: polman@amolf.nl. URL: http://www.erbium.nl.

${ }^{b}$ URL: http://www.wave-scattering.com

${ }^{c)}$ URL: http://www.colloid.nl.
}

as analytical calculations ${ }^{20}$ on prolate $\mathrm{Au}$ shell particles showed local field intensity enhancements of over three orders of magnitude at the particle tips.

The field enhancement resulting from collective plasmon modes in spherical and anisotropic core-shell colloids typically extends only several tens of nanometers from the surface of the particle. It is typically highest for small particles with core radius in the size range of $50-100 \mathrm{~nm}$. A further consideration in fluorescence enhancement experiments is that very close to the metal surface $(<10 \mathrm{~nm})$, where the field enhancements are highest, coupling to higher-order dark plasmonic modes introduces quenching paths, limiting the effect of plasmon-enhanced luminescence. ${ }^{6,20-23}$

While the collective scattering and absorption resonances due to the free electrons in the metal shell are now reasonably well studied, there is an additional resonance that has not received much attention. This is the geometric cavity mode that results from confinement of light inside the dielectric core, with the cavity boundaries determined by the metal shell. Teperik et al. ${ }^{24}$ performed calculations of the radiative decay rate and resonance frequencies of plasmons in metal nanoshells using a formalism developed earlier for dielectrics. ${ }^{25}$ They observed significantly reduced plasmon decay times at well-defined resonance wavelengths, with concomitant field enhancements inside the shell. In this paper we perform FDTD calculations ${ }^{26}$ analytical Mie theory, as well as calculations using the $T$-matrix method ${ }^{13,27}$ to fur- 
ther investigate the properties of this geometrical cavity mode. We study the cavity mode field distribution upon excitation and ringdown and determine the three-dimensional field distribution in the cavity. We determine how the resonance depends on geometric factors such as core diameter, shell thickness, refractive index, and shape anisotropy. We show that, depending on metal shell thickness, cavity quality factors $Q>150$ can be achieved. We compare the field enhancement and volume with other resonant plasmon geometries and derive the Purcell factor for this metal shell cavity mode.

\section{FDTD CALCULATION SCHEME}

Three-dimensional full-field electromagnetic simulations based on FDTD techniques ${ }^{28}$ were employed to study the electromagnetic field distributions of spherical and anisotropic Au shell colloids. The dielectric core (index $n=1.45$ ) has a radius $R_{\text {core }}=228 \mathrm{~nm}$ and is covered by a Au layer with thickness $t_{\mathrm{Au}}=38 \mathrm{~nm}$. These numbers are equal to those in experiments on the collective plasmon modes of core-shell particles reported earlier. ${ }^{15}$ The embedding medium is taken equal to that of the core material, so that the geometry simplifies to that of a $\mathrm{Au}$ shell embedded in a homogeneous medium. The optical response of $\mathrm{Au}$ is modeled using the Drude model,

$$
\varepsilon(\omega)=\varepsilon_{d}-\frac{\omega_{p}^{2}}{\omega^{2}+i \omega \gamma},
$$

with $\quad \varepsilon_{d}=9.54, \quad \omega_{p}=1.3 \times 10^{16} \mathrm{rad} / \mathrm{s}, \quad$ and $\quad \gamma=1.25$ $\times 10^{14} \mathrm{rad} / \mathrm{s}$, which provides a reasonably good fit to tabulated experimental data ${ }^{29}$ for photon energies smaller than $3 \mathrm{eV}$.

The particle is placed in the center of a threedimensional rectangular simulation box with dimensions about three times the particle size. For example, the box dimensions for a sphere with $R_{\mathrm{tot}}=266 \mathrm{~nm}$ were $1.3 \times 1.3$ $\times 1.3 \mu \mathrm{m}^{3}$, while for an oblate ellipsoid with an aspect ratio of 2.5 the simulation box measured $1.5 \times 1.5 \times 1.0 \mu \mathrm{m}^{3}$. In order to correctly represent the large field gradients near the curved metal surfaces, a linearly graded decrease in mesh size near the metal surface was used. Computer memory constrained the mesh to $3 \times 10^{6}$ grid cells. Consequently, for the large particles under consideration, the cells in the immediate vicinity of the particle had a width of about $7 \mathrm{~nm}$ and those on the outer boundary of the simulation volume had widths of about $35 \mathrm{~nm}$. The simulation results were found to be robust against small changes in mesh cell density. For ellipsoidal particles, the incident light was linearly polarized along one of the main axes of the particle.

A two-step process was used to determine the particle's resonant mode and its frequency. First, the simulation volume is illuminated by a plane wave with an off-resonance frequency, allowing the particle to become polarized and store energy. The plane wave was gradually switched on in approximately three optical cycles. After steady state was reached, the incident field is switched off and the electric field amplitude is monitored in the time domain as the particle modes excited by the incident plane wave decay (ring down). This signal is transformed into the frequency domain by a fast Fourier transform (FFT) to provide spectral characteristics of the particle resonances. Once the resonance frequencies are determined in this way, on-resonance excitation is used to excite individual modes to examine the corresponding spatial distribution of the field intensity. Such distributions can be used to discriminate between spectral features that correspond to physical resonances and unphysical artifacts of the simulation or of the frequency domain transform. For example, small "hot spots" can occur in the intensity maps as a result of faceted corners in the rendering of the curved surfaces. Additionally, the on-resonance field intensity distribution allows visualization and quantification of the local field enhancement in the nanoparticle.

\section{SPHERICAL AU SHELL CAVITIES}

Figure 1 shows the different steps in the FDTD calculation for a Au shell with $R_{\text {core }}=228 \mathrm{~nm}$ and $t_{\mathrm{Au}}=38 \mathrm{~nm}$. A snapshot in time of the $x$-component of the electric field is shown in Fig. 1(a) for an incident plane wave at an offresonance frequency of $150 \mathrm{THz}$ (free space wavelength $\lambda$ $=2.0 \mu \mathrm{m})$. The image shows the plane that contains both the propagation direction of the plane wave $(y$, indicated by the vertical arrow) and the polarization ( $x$, indicated by the horizontal arrow). Close to the outer metal surface, the amplitude of the electric field is enhanced by about a factor of 3 . The field inside the shell is about one-tenth of that of the incoming plane wave.

Figure 1(b) shows the time evolution of the electric field amplitude in a monitor point at the center of the particle. The incident plane wave is gradually switched on at $t=-40 \mathrm{fs}$ and switched off at $t=0$, whereupon the particle's relaxation is observed. The FFT of the decay trace is shown in Fig. 1(c) and reveals a main resonance peak at $335 \mathrm{THz}(\lambda$ $=895 \mathrm{~nm}$ ). From the temporal decay of the electric field intensity inside the dielectric core, the quality factor for this cavity mode is found to be $Q=2 \pi f \tau=35$, with $f$ as the frequency and $\tau$ as the decay time, respectively.

Since absorption, scattering, and ringdown are resonant phenomena, the frequency at which the peak FFT response occurs is directly comparable to the frequency of maximum extinction in an optical spectrum. Figure 2 shows the extinction spectrum of the Au shell particle, as calculated by Mie theory. ${ }^{30}$ The separate contributions of absorption and scattering are shown as well. The spectra show that the extinction for this particle is mainly due to scattering. The extinction contributions of dipole $(D)$, quadrupole $(Q)$, and octupole $(O)$ collective modes, described in detail in Ref. 16, are indicated separately in the figure. Tabulated optical constants for Au by Johnson and Christy ${ }^{29}$ were used for the calculation of these spectra. Therefore, the contribution of interband transitions at wavelengths smaller than $600 \mathrm{~nm}$ is also observed in the absorption spectrum. These are absent in the FDTD calculation of Fig. 1(c) as that employed a Drude model for the dielectric constants.

The extinction spectrum for the dipolar mode in Fig. 2 shows a distinct peak at $885 \mathrm{~nm}(340 \mathrm{THz}$, indicated by the arrow). This resonance wavelength is in good correspon- 

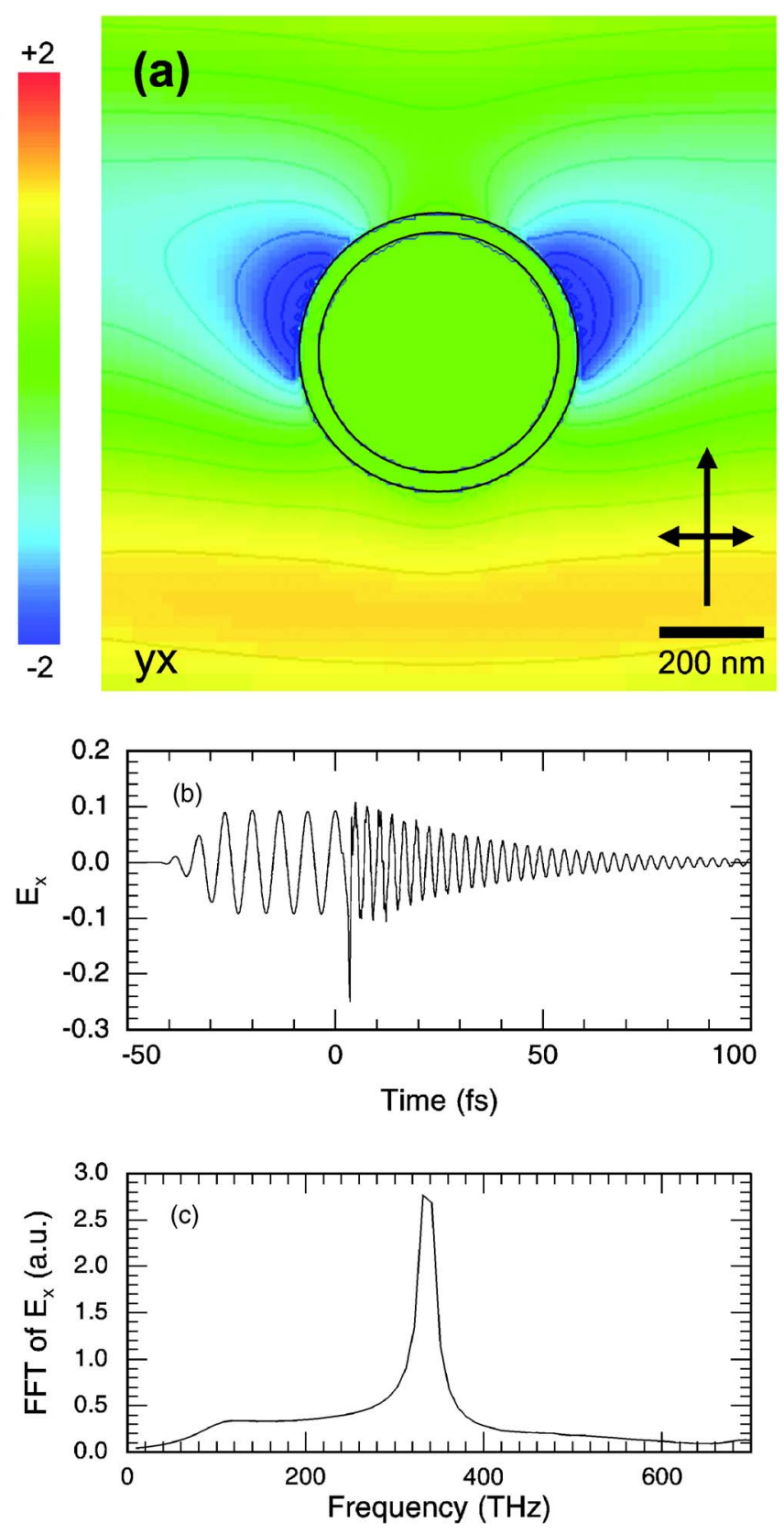

FIG. 1. (Color online) (a) FDTD calculation snapshot [at $t=10$ in Fig. 1(b)] of the $x$-component of the electric field of a plane wave propagating in the $y$-direction, at a frequency of $150 \mathrm{THz}$, and interacting with a spherical $\mathrm{Au}$ shell $\left(R_{\text {core }}=228 \mathrm{~nm}, t_{\mathrm{Au}}=38 \mathrm{~nm}\right)$ in silica $(n=1.45)$. The plane wave is polarized in the plane ( $x$-direction). The two concentric circles indicate the inner and outer boundaries of the Au shell. Amplitude is normalized to peak amplitude of the incident wave. The color scale is saturated at intensity 2 . (b) The excitation and relaxation traces of the $x$-component of the electric field in a monitor point in the center of the particle. The plane wave is gradually turned on a $t=-40 \mathrm{fs}$ and turned off at $t=0$. (c) FFT of the decay of the $x$-component of the electric field, showing a resonance peak at a frequency of $335 \mathrm{THz}$.

dence with the resonance frequency at $335 \mathrm{THz}$ observed in the ringdown of the FDTD calculation [Fig. 1(c)]. This indicates that the extinction resonance at $885 \mathrm{~nm}$ is due to a cavity mode coupled to the dipolar collective mode.

The field distribution of the resonant mode is investigated with on-resonance plane-wave excitation at $335 \mathrm{THz}$. A time snapshot under steady state is shown in Fig. 3 for a time at which the electric field inside and close to the particle
Frequency $(\mathrm{THz})$

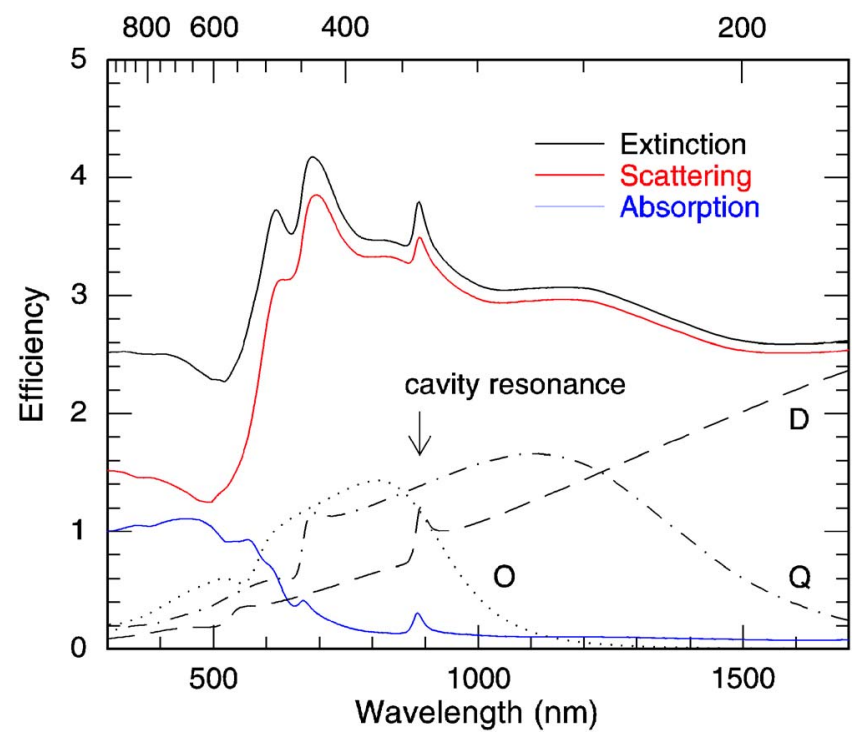

FIG. 2. (Color online) Mie theory calculation of extinction (black), scattering (red), and absorption (blue) efficiency spectra of a Au shell in a silica matrix $\left(R_{\text {core }}=228 \mathrm{~nm}, t_{\mathrm{Au}}=38 \mathrm{~nm}\right)$. The dipolar $(D$, dashed $)$, quadrupole ( $Q$, dash dotted), and octupole ( $O$, dotted line) mode contributions to the extinction are indicated in the figure. The cavity resonance peak at $885 \mathrm{~nm}$ is indicated by an arrow.

is at a maximum. Data are shown for the $x$-component of the electric field $[(\mathrm{a})-(\mathrm{c})]$ and the time-averaged intensity $[(\mathrm{d})-$ (f)], all normalized to the incident field. Data are shown in the $y x$ plane (spanning propagation and polarization direction), the $y z$ plane (perpendicular to polarization direction), and the $z x$ plane (perpendicular to propagation direction).

The images in Fig. 3 clearly reveal how the incoming plane wave interacts with the Au shell. The phase fronts of the plane wave can be observed at the boundaries of the simulation volume in the $y x$ and $y z$ planes. Time series of the snapshots of the $y x$ plane [as in Fig. 3(a)] show propagation of the electric field phase front along the outer surface of the metal. It is clearly seen that the electric field inside the dielectric core oscillates out of phase relative to the field outside the shell [see, e.g., Fig. 3(c)]. Figures 3(d)-3(f) show the time-averaged field intensity inside corresponding to the data in Figs. 3(a)-3(c). The maximum field enhancement within the core amounts to a factor of 6.5. The field enhancement is the net result of the effect of cavity confinement $(Q=35)$ and (limited) incoupling through the $38 \mathrm{~nm}$ thick Au shell.

For a better understanding of the cavity mode, Mie theory calculations were exploited to study the dependence of the resonance frequency on core diameter, shell thickness, as well as the dielectric constants of core and surrounding medium. Figure 4(a) shows calculations of the extinction efficiency (defined as extinction cross section relative to geometrical cross section) for core indices in the range $n$ $=1.1-1.5\left(R_{\text {core }}=228 \mathrm{~nm}, t_{\mathrm{Au}}=35 \mathrm{~nm}\right)$. A large effect of refractive index on the resonance wavelength is observed. The redshift is approximately linear with index, clearly reflecting the geometrical cavity nature of the mode, and amounts to $\sim 550 \mathrm{~nm}$ per refractive index unit.

Varying the dielectric constants of the surrounding me- 
Ex
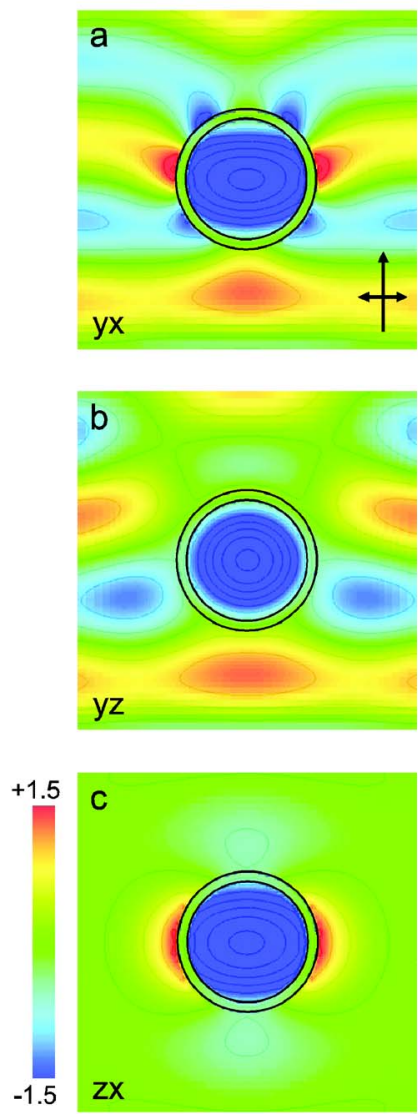
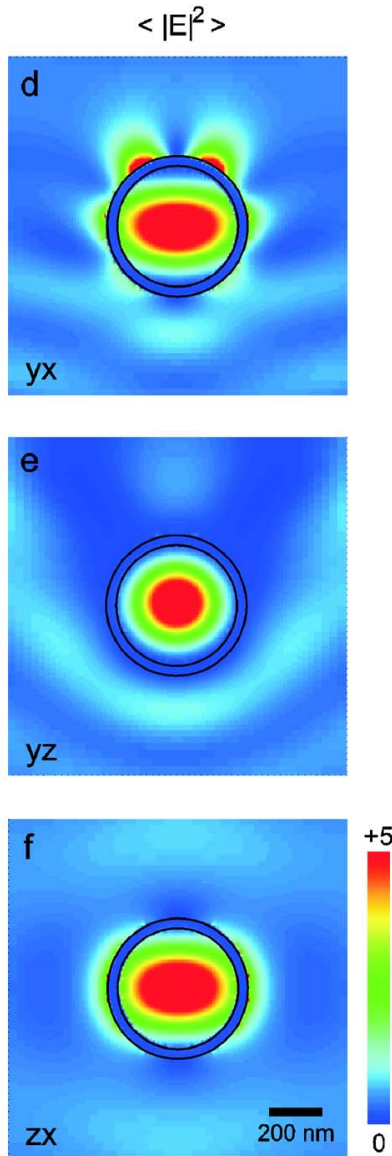

FIG. 3. (Color online) FDTD time snapshot of the $x$-component (left) and time-averaged intensity (right) of the electric field in [(a) and (d)] the $y x$ plane [(b) and (e)] the $y z$ plane, and [(c) and (f)] the $z x$ plane for a spherical Au shell colloid in silica $\left(R_{\text {core }}=228 \mathrm{~nm}, t_{\mathrm{Au}}=38 \mathrm{~nm}\right)$. The particle was excited on-resonance with a plane wave at $335 \mathrm{THz}$. The plane wave propagates in the $y$-direction and is polarized along $x$. In (d) and (f) the color scale is saturated at 5.0; the peak intensity inside the core is 6.5 .

dium did not result in a significant shift in the resonance wavelength (data not shown). This demonstrates that the resonance properties of the cavity mode are set by the continuity conditions of Maxwell's equations at the core/shell boundary and not at the boundary between the shell and the surrounding medium. Note that for the highest index in Fig. 4 , a second peak appears in absorption, at $\sim 680 \mathrm{~nm}$. We attribute this to a cavity mode coupled to a collective quadrupole mode, as a resonance near $680 \mathrm{~nm}$ is also observed in the extinction spectrum for the quadrupole mode in Fig. 2. Given the relatively small core radius, this mode only appears for sufficiently high index and correspondingly small effective wavelength inside the core.

Figures 4(b) and 4(c) show the dependence of the cavity resonance spectrum on core/shell dimensions (embedding index $n=1.45)$. In Fig. 4(b) the core radius is fixed at $228 \mathrm{~nm}$ and the total particle radius varied in the range of 260-280 nm; in Fig. 4(c) the total particle radius is fixed at $266 \mathrm{~nm}$ while the core radius varied in the range of $220-235 \mathrm{~nm}$. In both cases, the metal shell thickness varied in the range of $\sim 30-50 \mathrm{~nm}$. Figure 4(b) shows a slight blueshift of the resonance at $890 \mathrm{~nm}$ for increasing shell thickness. We attribute this to a gradual phase shift upon increased

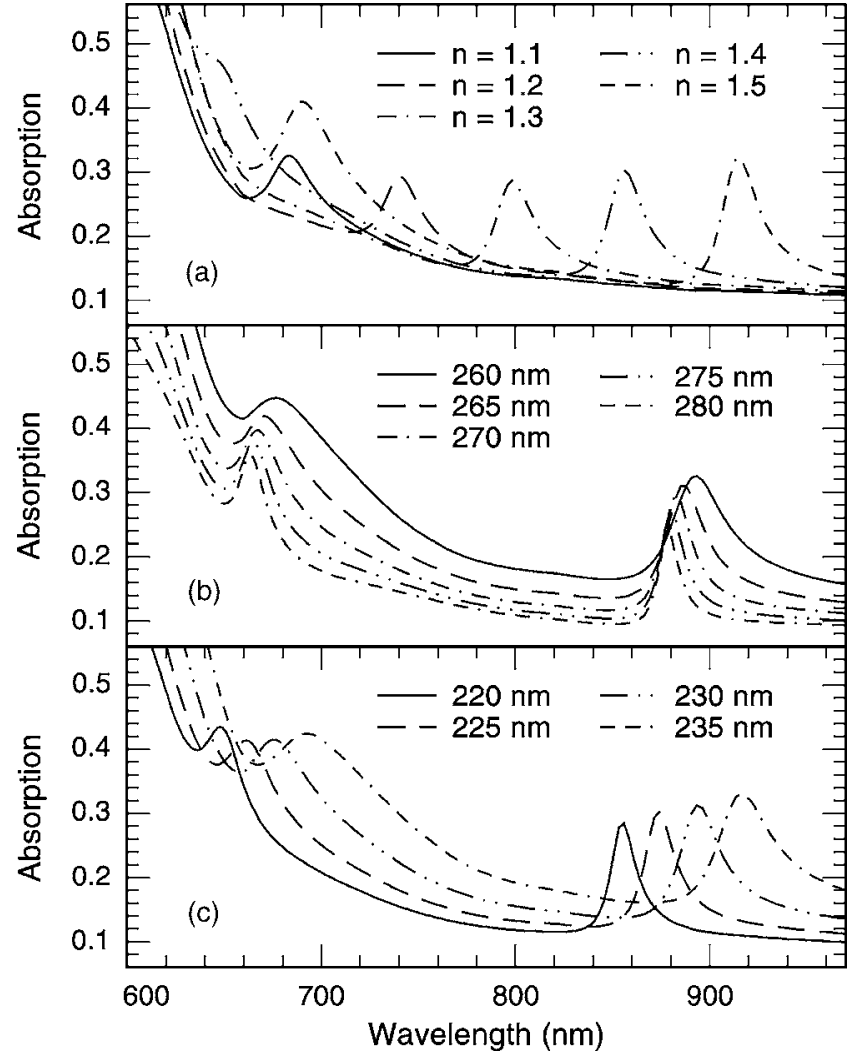

FIG. 4. Mie theory calculations of optical absorption efficiency spectra for spherical core/shell colloids embedded in a medium with $n=1.45$ (a) for different refractive indices of the core $\left(R_{\text {core }}=228 \mathrm{~nm}, t_{\mathrm{Au}}=38 \mathrm{~nm}\right)$, (b) for different total radii at fixed core radius of $228 \mathrm{~nm}\left(n_{\text {core }}=1.45\right)$, and (c) as a function of core radii at a fixed total radius of $266 \mathrm{~nm}\left(n_{\text {core }}=1.45\right)$. Shell thickness varies in the range of $30-50 \mathrm{~nm}$ in (b) and (c).

confinement of the cavity mode at the core-shell interface, as the metal thickness increases. Indeed, as Fig. 4(b) shows, for largest metal thickness, extending beyond the skin depth, the resonance shift converges. The figure also shows a progressive resonance peak narrowing upon increasing the shell thickness, in agreement with the stronger cavity confinement at larger metal thickness. Note that the higher-order mode at $\sim 680 \mathrm{~nm}$ shows the same trends of spectral shift and narrowing with increasing shell thickness as the fundamental mode.

Figure 4(c) shows a large resonance redshift as the core radius is increased. The linear shift with $R_{\text {core }}$ is in agreement with the geometrical nature of the cavity mode. Here too, a progressive resonance peak narrowing is observed upon decreasing $R_{\text {core }}$, corresponding to an increased Au shell thickness. For shell thicknesses well beyond the skin depth the shell effectively acts as a closed cavity with losses only determined by reflection losses and not by radiation losses into the far field. The finite resonance width is then determined by losses in the metal that are included in the calculation through the imaginary part of the dielectric constant.

Using the data in Fig. 4(b) and additional data for larger total radius, we determine the relation between cavity quality factor and metal shell thickness. The quality factor is determined from the resonance linewidth $(Q=\Delta \lambda / \lambda)$ determined from Lorentzian fits to absorption spectra plotted in the inset of Fig. 5. For shell thickness below $30 \mathrm{~nm}$, linewidths could 


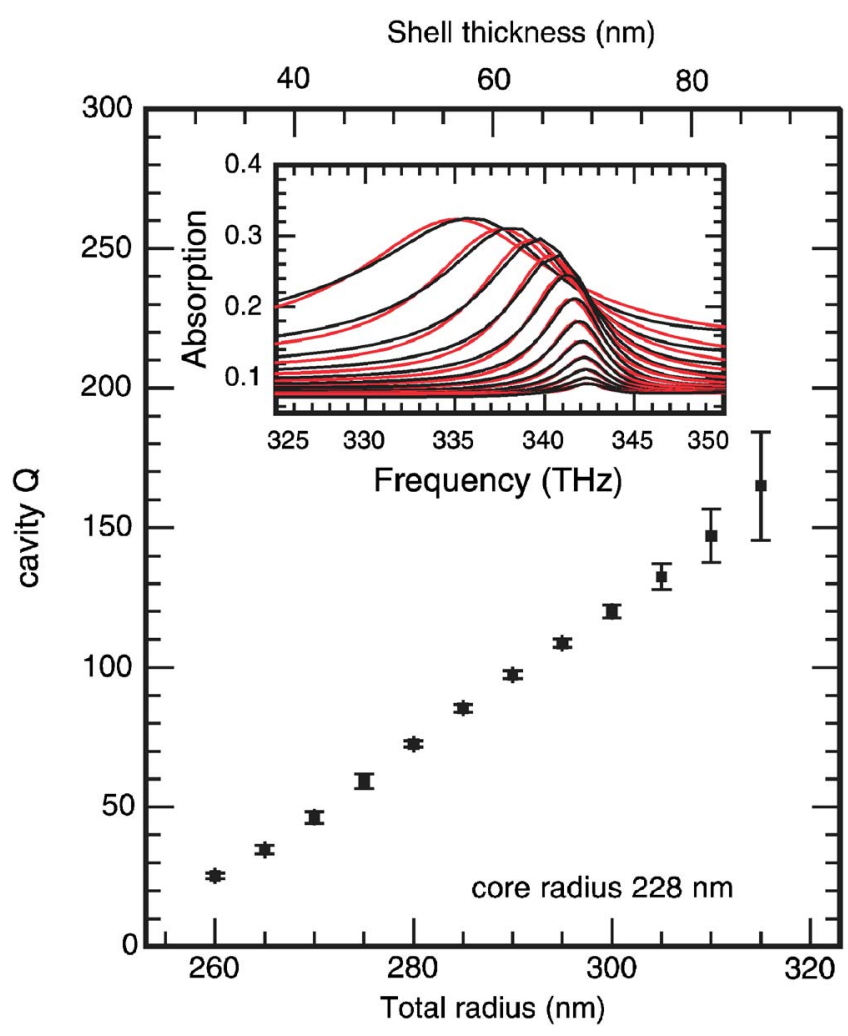

FIG. 5. (Color online) Cavity quality factor as a function of total particle radius for a Au shell with fixed $R_{\text {core }}=228 \mathrm{~nm}(n=1.45)$. The corresponding metal shell thickness is plotted on the top scale. The inset shows the absorption spectra from which the quality factor was derived (drawn lines, calculations; dashed lines: Lorentzian fits).

not be reliably determined as the broad resonance spectrum is superimposed on the absorption background of the collective plasmon modes (see Fig. 2). For shell thickness $>90 \mathrm{~nm}$, the resonance peak is very weak (i.e., coupling of the incident plane wave into the cavity mode is very small) and numerical limitations make it impossible to derive reliable linewidths. Overall, Fig. 5 shows that cavity quality factors $Q>150$ can be achieved in these Au shell colloids. We note that these quality factors are more than ten times larger than those of the collective resonance modes that show only very broad spectral features (see Fig. 2). Indeed, our earlier calculations ${ }^{31}$ have shown characteristic dephasing times for the collective modes of core-shell colloids in the range $T_{2}=1-5 \mathrm{fs}$, much shorter than the relaxation time observed in the ringdown in Fig. 1(b). The $Q$ value found here is larger than that found in Ref. 24, which we attribute due to a difference in the used dielectric constants for $\mathrm{Au}$.

To take advantage of these high- $Q$ modes for fluorescence or Raman enhancement, the particle core must be accessible from the outer world. Experiments have been performed to dissolve the inner silica shell in $\mathrm{HF}^{32}$ and show that most of the silica core can be dissolved while the particles continue to behave optically as closed shells. This effect is ascribed to nanoscale porosity of the thin metal shell. This technique makes the field inside of the metal shells accessible for experiments that take advantage of the enhanced local field.

\section{SPHERICAL CORE-SHELL CAVITIES: FIELD ENHANCEMENT, MODE VOLUME, AND PURCELL EFFECT}

For practical use of these core-shell cavity resonances in fluorescence enhancement or Raman scattering, both the field enhancement and the volume in which the field is enhanced are important. We compare the data found in this paper to those found for solid metal nanoparticles. As noted in the Introduction, solid metal particles can show 100-fold field enhancements over very small distances near the metal surface. Anisotropically shaped bulk metal particles can have local hot spots with over 1000-fold field enhancement, ${ }^{33}$ but only in nanoscale volumes. For fluorescence enhancement applications, quenching close to the metal surface must be taken into account, reducing the effective volume of field enhancement.

The core-shell cavity resonances found here have smaller field enhancement (e.g., factor of 6.5 in Fig. 3), but in a much larger volume. Overall, the integral of field enhancement over mode volume is higher for optimized coreshell cavities than for optimized bulk metal nanoparticles, making the core-shell colloids ideally suited for applications in fluorescent and Raman enhancement. An optimized coreshell geometry for these applications should be engineered such that critical coupling occurs, ${ }^{34}$ i.e., that the coupling rate into the cavity (through the metal shell) equals the cavity loss rate.

While the mode volume in the core-shell colloids can be considered relatively large by the above arguments, it is small when compared to several other optical microcavity geometries. The mode volume in the core-shell colloids studied here is $V=0.2(\lambda / n)^{3}$, which is similar to the mode volume in, e.g., one-dimensional Fabry-Pérot cavities and, 1001000 times smaller than that in microdisk or microtoroid whispering gallery mode cavities. ${ }^{35}$ It is comparable to that of the smallest photonic crystal cavities. Mode volume $V$, cavity $Q$, and cavity geometry are critical parameters determining the spontaneous emission rate and lasing threshold in optical microcavities. A characteristic Purcell factor for the present core-shell cavities is

$$
P=\frac{3}{4 \pi^{2}}\left(\frac{\lambda}{n}\right)^{3} \frac{Q}{V}=54,
$$

assuming a homogeneous field distribution in the cavity core, $Q=150$ and $\lambda=895 \mathrm{~nm}$. Taking into account the inhomogeneous field distribution as in Fig. 3(f), an even higher local value for $P$ is expected. This again shows that very large spontaneous emission enhancements are expected for optical emitters placed inside the cavity. Note that, as a result of the relatively modest $Q$, not very stringent requirements are put on the emission linewidth of the emitter in order to observe the Purcell effect in these core-shell cavities.

\section{OBLATE ELLIPSOIDAL AU SHELL COLLOIDS}

As described in the Introduction, shape anisotropy is an interesting parameter to control core/shell particle resonant behavior. Experiments and theory on anisotropically shaped core-shell particles have shown large blue- and red shifts of 


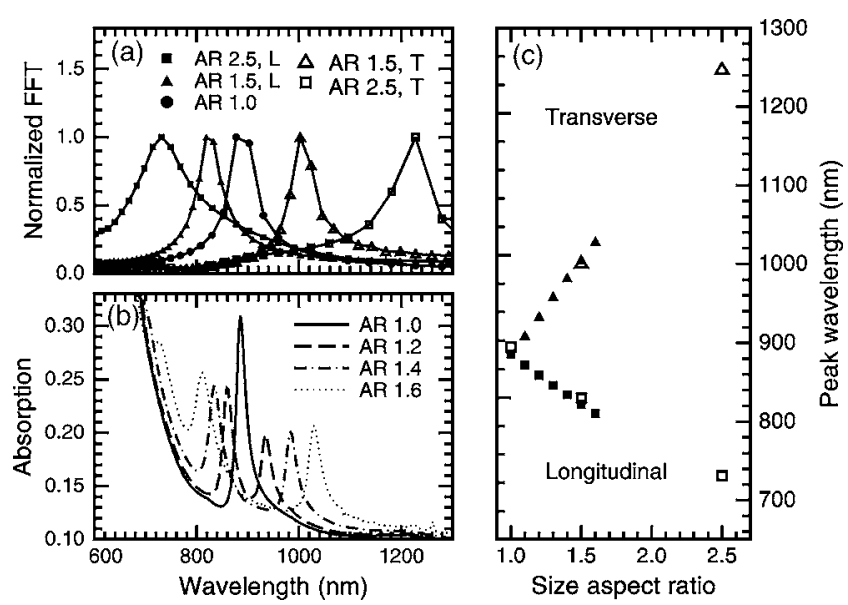

FIG. 6. Resonance spectra of oblate ellipsoidal Au shells in silica with volume equivalent to a sphere with $R_{\text {core }}=228 \mathrm{~nm}, t_{\mathrm{Au}}=38 \mathrm{~nm}$. (a) Normalized FFT results from FDTD calculations for aspect ratios of 1.0, 1.5, and 2.5 for polarizations parallel to the major $(L)$ and minor $(T)$ axes of the colloid. (b) Orientation-averaged absorption efficiency for aspect ratios of 1.0 (solid line), 1.2 (dashed line), 1.4 (dash-dotted line), and 1.6 (dotted line) calculated by the $T$-matrix method. (c) Resonance peak wavelength as a function of size aspect ratio for FDTD (open) and $T$-matrix calculations (closed symbols).

longitudinal and transverse collective dipolar and higherorder modes in scattering, respectively. ${ }^{12,14,15}$ It is therefore interesting to study the dependence of the geometrical cavity modes on anisotropy.

FDTD calculations were performed on oblate ellipsoidal shells, defined by two concentric oblate ellipsoids (representing the shell's inner and outer boundary), both with identical aspect ratio. Core and shell volume were taken equal to that of a spherical Au shell particle with $R_{\text {core }}=228 \mathrm{~nm}$ and $t_{\mathrm{Au}}$ $=38 \mathrm{~nm}$, as in Figs. 1-5. Figure 6(a) shows the normalized FFT spectra for particles with size aspect ratios of 1.0, 1.5, and 2.5. The spectrum of the spherical shell shows only one cavity resonance at $895 \mathrm{~nm}(335 \mathrm{THz}$, closed spheres, as in Fig. 1), whereas the cavity resonance for the anisotropic particles is split. For light incident along the minor axis, with polarization along a major (longitudinal, $L$ ) axis of the oblate ellipsoidal Au shell, a blueshift is observed: the resonance shifts to 830 and $730 \mathrm{~nm}$ for aspect ratios of 1.5 (triangles) and 2.5 (squares), respectively. For light polarized along the minor (transverse, $T$ ) axis, the resonances are redshifted to 1000 and $1250 \mathrm{~nm}$, respectively. The relative redshift is twice as large as the relative blueshift, as expected to first order for an oblate geometry with constant volume. Note that the resonance shifts with anisotropy show an opposite trend as that observed for the collective dipolar and higher-order plasmon modes. ${ }^{15}$ It is in agreement with the trend observed for a quasistatic calculation for an ellipsoidal void in bulk metal, which shows blueshifted longitudinal and redshifted transverse resonant modes for increasing anisotropy. ${ }^{13}$

The optical absorption and scattering spectra of the oblate ellipsoidal metal shells were also calculated using the $T$-matrix method, ${ }^{36,37}$ with a recent improvement. ${ }^{38}$ Figure 6(b) shows the absorption efficiency spectrum averaged over the orientation of the particle, for particles with aspect ratios ranging from 1.0 to 1.6. The resonance peak splits for spheroidal particles, again with a larger relative shift for the peak shifting to the red than for the one shifting to the blue, as also observed in the FDTD results in Fig. 6(a). The total strength of the absorption feature (integrated over frequency) is similar to that for ellipsoidal particles as for spheres. The resonance wavelengths for transverse and longitudinal modes obtained by the two methods are summarized in Fig. 6(c) as a function of aspect ratio. The results for both methods are in good agreement and show that a large degree of wavelength tunability is achievable upon a change in aspect ratio at constant volume. It also demonstrates that the ringdown technique provides an accurate description of cavityresonant behavior, also for nonspherical shapes.

\section{CONCLUSIONS}

Spherical core-shell colloids composed of a dielectric core and a $\mathrm{Au}$ shell, with dimensions comparable to the wavelength of light, exhibit geometrical cavity resonances that depend on the size, shape, and dielectric constant of the dielectric core and thickness of the metal shell. Using Mie calculations we have determined the field enhancement and cavity quality factor as a function of thickness of the metal shell. Cavity quality factors $Q>150$ are observed, with over fivefold field enhancements over a large fraction of the volume of the core. Due to the small cavity mode volume, $V$ $=0.2(\lambda / n)^{3}$, a large Purcell factor $P=54$ is found. Mie calculations as well as $T$-matrix calculations for oblate ellipsoidal core-shell colloids reveal splitting of the cavity resonance wavelength, with a blueshifted longitudinal and a redshifted transverse mode. The calculated data demonstrate the feasibility of using spherical as well as ellipsoidal $\mathrm{Au}$ shell colloids in nanoscale light sources, sensors, or lasers.

\section{ACKNOWLEDGMENTS}

Femius Koenderink (FOM-AMOLF) and Sébastien Bidault (FOM-AMOLF) are acknowledged for stimulating and helpful discussions. This work is part of the research program of the "Stichting voor Fundamenteel Onderzoek der Materie (FOM)," which is financially supported by the "Nederlandse organisatie voor Wetenschappelijk Onderzoek (NWO)."

${ }^{1}$ J. Gersten and A. Nitzan, J. Chem. Phys. 73, 3023 (1980).

${ }^{2}$ J. S. Biteen, N. S. Lewis, H. A. Atwater, H. Mertens, and A. Polman, Appl. Phys. Lett. 88, 131109 (2006).

${ }^{3}$ M. Moskovit, Rev. Mod. Phys. 57, 783 (1985).

${ }^{4}$ J. B. Jackson and N. J. Halas, Proc. Natl. Acad. Sci. U.S.A. 101, 17930 (2004).

${ }^{5}$ A. Parfenov, I. Gryczynski, J. Malicka, C. D. Geddes, and J. R. Lakowicz, J. Phys. Chem. B 107, 8829 (2003).

${ }^{6}$ A. Moroz, Chem. Phys. 317, 1 (2005).

${ }^{7}$ O. G. Tovmachenko, C. Graf, D. J. van den Heuvel, A. van Blaaderen, and H. C. Gerritsen, Adv. Mater. (Weinheim, Ger.) 18, 91 (2006).

${ }^{8}$ J. Enderlein, Appl. Phys. Lett. 80, 315 (2002).

${ }^{9}$ J. Enderlein, Phys. Chem. Chem. Phys. 4, 2780 (2002).

${ }^{10}$ A. L. Aden and M. Kerker, J. Appl. Phys. 22, 1242 (1951).

${ }^{11}$ A. E. Neeves and M. H. Birnboim, J. Opt. Soc. Am. B 6, 787 (1989).

${ }^{12}$ S. J. Oldenburg, R. D. Averitt, S. L. Westcott, and N. J. Halas, Chem. Phys. Lett. 288, 243 (1998).

${ }^{13}$ C. F. Bohren and D. R. Huffman, Absorption and Scattering of Light by Small Particles (Wiley, New York, 1983).

${ }^{14}$ H. Wang, D. W. Brandl, F. Le, P. Nordlander, and N. J. Halas, Nano Lett. 6, 827 (2006).

${ }^{15}$ J. J. Penninkhof, C. Graf, T. van Dillen, A. M. Vredenberg, A. van Blaa- 
deren, and A. Polman, Adv. Mater. (Weinheim, Ger.) 17, 1484 (2005).

${ }^{16}$ J. J. Penninkhof, A. Moroz, A. van Blaaderen, and A. Polman, J. Phys Chem. C 112, 4146 (2008).

${ }^{17}$ S. J. Oldenburg, S. L. Westcott, R. D. Averitt, and N. J. Halas, J. Chem. Phys. 111, 4729 (1999).

${ }^{18}$ J. B. Jackson, S. L. Westcott, L. R. Hirsch, J. L. West, and N. J. Halas, Appl. Phys. Lett. 82, 257 (2003).

${ }^{19}$ H. Wang, D. W. Brandl, F. Le, P. Nordlander, and N. J. Halas, Nano Lett. 6, 827 (2006).

${ }^{20} \mathrm{H}$. Mertens and A. Polman, a code is available on www.erbium.nl

${ }^{21}$ P. F. Liao and A. Wokaun, J. Chem. Phys. 76, 751 (1982).

${ }^{22}$ E. Dulkeith, A. C. Morteani, T. Niedereichholz, T. A. Klar, J. Feldmann, S. A. Levi, F. C. J. M. van Veggel, D. N. Reinhoudt, M. Möller, and D. I. Gittins, Phys. Rev. Lett. 89, 203002 (2002).

${ }^{23}$ H. Mertens, A. F. Koenderink, and A. Polman, Phys. Rev. B 75, 115123 (2007).

${ }^{24}$ T. V. Teperik, V. V. Popov, and F. J. García de Abajo, Phys. Rev. B 69 , 155402 (2004).

${ }^{25}$ F. J. García de Abajo, Phys. Rev. B 60, 6086 (1999).
${ }^{26}$ A. Taflove and S. C. Hagness, Computational Electrodynamics: The Finite-Difference Time Domain Method (Artech House, Norwood, MA, 2000).

${ }^{27}$ M. I. Mishchenko and L. D. Travis, J. Quant. Spectrosc. Radiat. Transf. 60, 309 (1998).

${ }^{28}$ Maxwell's equations by Finite Integration Algorithm (MAFIA) software, 4th ed., Version 4.106, CST, Darmstadt, Germany.

${ }^{29}$ P. B. Johnson and R. W. Christy, Phys. Rev. B 6, 4370 (1972).

${ }^{30} \mathrm{~A}$ code is available on http://www.wave-scattering.com//

${ }^{31} \mathrm{~A}$. Moroz (unpublished).

${ }^{32}$ C. Graf and A. van Blaaderen, Langmuir 18, 524 (2002).

${ }^{33}$ H. Mertens, A. F. Koenderink, and A. Polman, Phys. Rev. B 75, 115123 (2007).

${ }^{34}$ M. Cai, O. Painter, and K. J. Vahala, Phys. Rev. Lett. 85, 74 (2000).

${ }^{35}$ See K. J. Vahala, Nature (London) 424, 839 (2003) and references therein. ${ }^{36}$ M. I. Mishchenko, Appl. Opt. 39, 1026 (2000).

${ }^{37}$ A. Quirantes, J. Quant. Spectrosc. Radiat. Transf. 63, 263 (1999).

${ }^{38}$ A. Moroz, Appl. Opt. 44, 3604 (2005). 